\title{
A Brief Review of the Large Eddy Simulation Method
}

\author{
Sachin H Shinde ${ }^{1}$, Mrs. B. S. Shetty ${ }^{2}$ \\ M. Tech in Computer Science and Engineering (Specialization-Information Technology), WCE, Sangli ${ }^{1}$ \\ Assistant Professor, WCE, Sangli ${ }^{2}$
}

\begin{abstract}
Large-eddy simulation (LES) was originally proposed for simulating atmospheric flows in the 1960s and has become one of the most promising and successful methodology for simulating turbulent flows with the improvement of computing power. It is now feasible to simulate complex engineering flows using LES. However, apart from the computing power, significant challenges still remain for LES to reach a level of maturity that brings this approach to the mainstream of engineering and industrial computations. This paper will describe briefly overview of LES formalism first, present a quick glance at its history, review its current state focusing mainly on its applications in transitional flows and gas turbine combustor flows, discuss some major modeling and numerical challenges/issues that we are facing now and in the near future, and finish with the concluding remarks.
\end{abstract}

Keywords: Parallel computing, atmospheric flow, turbulent flow, cloud physics.

\section{INTRODUCTION}

Turbulence can be characterized as a presence of largely random fluctuations of velocity superposed on an otherwise an orderly mean flow. It is inherently unsteady with components in all directions. On the other hand, laminar flow is a phenomenon where air, gas, or a liquid flows in parallel layers and there is only a molecular mixing of layers. It is the opposite of turbulent flow, where the field elements are constantly mixing and moving in varied ways across a space. The most accurate description of turbulent flows is the Direct Numerical Simulation (DNS). Here, the approach is to solve the full Navier-Stokes equations. The goal is to capture all the scales that are present in a given flow, from the smallest to the largest eddies. This implies applying time-accurate computation on extremely fine grids, dictating the use of very small time steps, as well as the need to account for the three-dimensionality of turbulence. At present, this requirement limits the applicability of the method to low Reynolds number flows over simple geometry $[1,2]$.

In many cases, one is mainly interested in the steady-state fluid flow. By taking the time-average of the full NavierStokes equations, a set of equations with a less demand on computing resources can be obtained. This is the basis for the Reynolds-Averaged Navier-Stokes (RANS). In that approach, one solves only for the averaged quantities, while the turbulence itself is modeled separately. In practice, this has alleviated the need for accurate representation of the eddies in the flow, and only taking the average of their effects on the mean flow. This approach has been the backbone in the industrial (Computational Fluid Dynamics) CFD applications for the last few decades, due to its modest computing requirement.

Several turbulence models are widely available for use with this approach. For several types of flows, however, knowledge of the transient behavior of the flow is necessary, to study the dynamic interaction between turbulence and the mean flow, or to understand the structure of the turbulence itself. For these, the RANS method may not be sufficient, due to the fact that averaging approach ignores some main characteristics of the time-dependent solutions. In these cases, the Large-Eddy Simulation (LES) may come as an excellent method of choice. In essence, LES recognizes that the behavior of the vertical structures in turbulence depends largely on their sizes. LES seeks to accurately capture the larger eddies, while only modeling the smaller ones. It has been assumed that at small length scale, the eddies are more-or-less uniform. Most of the research taking place in CFD is dealing with turbulent flows. Since it can be said that in nature the majority of flows are turbulent, it is an essential task to model this kind of flows. To achieve this goal, many models have been developed to represent solutions for turbulent flows. As mentioned above, the three main approaches developed so far are the direct numerical simulation (DNS), the Reynolds-averaged solutions (RANS) and the large eddy simulation (LES). Each of these approaches can be used for a certain and specific problems [3].

The DNS approach is an accurate solution of Navier-Stokes equations. In this method the spatial and temporal scales of turbulence will be resolved. The main purpose of this model is to solve turbulent velocity field, without a use of any modeling, Therefore, the Navier-Stokes equations will be solved accurately which requires that the grids should be very _ne and the time steps need to be very short. This task needs long time for calculations and requires powerful computers and it is limited to a low Reynolds number [4]. 
Vol. 6, Issue 5, May 2017

\section{RELATED WORKS}

\subsection{LES with WRF}

The Weather Research and Forecasting model (WRF) is a state of the art atmospheric modeling system that can be employed to simulate atmospheric flow over a wide range of scales spanning numerical weather prediction (NWP) to large-eddy simulation (LES). Historically, WRF has been used primarily to simulate NWP-scale features on computational meshes with horizontal sizes $(\Delta \mathrm{h})$ of several kilometers. Increasingly, WRF is being run at scales fine enough to resolve explicitly the important scales of three-dimensional atmospheric turbulence, with horizontal scales less than 100 meters. Atmospheric turbulence encompasses many different scales which can interact significantly and in nonlinear ways: energy can propagate both up and down scale. Large scale simulations ( $\mathrm{h} \sim 10 \mathrm{~s}$ of $\mathrm{km}$ ) use grids that are sufficiently coarse so that no scales associated with atmospheric turbulence are representable on the computational mesh. In such cases, the grid cell average velocity predicted on the nodes comprising the mesh represents the slowlyvarying or "mean" state of the flow. Since motions on the scale of atmospheric turbulence cannot be resolved, their influence on the slowly-varying component must be modeled. Typically the horizontal mixing is specified using the deformation of the large-scale flow, while the vertical mixing is prescribed in a so-called planetary boundary layer (PBL) parameterization. Such parameterizations utilize assumptions appropriate for large scales to provide a representation of the gross effects the entire turbulence spectrum on the evolution of the slowly-varying component of the flow field represented by the field variables on the computational mesh [5]. The LES, in contrast, is a technique in which the turbulence-generating motions within a turbulent flow field are explicitly resolved, while the smaller-scale portion of the turbulence spectrum is removed from the field using a spatial filter. The effects of the filtered scales of motion on the explicitly resolved turbulence field are modeled in a sub filter-scale (SFS) stress model.

\subsection{LES with PALM}

PALM is a large-eddy simulation (LES) model for atmospheric and oceanic flows which is especially designed for performing on massively parallel computer architectures. PALM is free software. It can be redistributed and/or modified under the terms of the GNU General Public License (v3).First version is released in 1997, continuously developed. Written in FORTRAN 95, very high performance and scalability on all state-of-the-art parallel architectures. It has one code for atmosphere and other for ocean. PALM Scripts allow for very comfortable operation of the model can be adapted for any kind of (UNIX) system by simply adjusting a configuration file. Batch jobs and job chains (restart mechanism) can be automatically created (requires manual adjustments of scripts). Runs are controlled by a simple NAMELIST parameter file Error messages inform in case of parameter inconsistencies or other problems detected during runtime. Standard data analysis is done within the simulation itself. All output is in standard netCDF data format.Parallelized LES Model (PALM) is based on the non-parallelized LES code described by Raasch and Etling (1991). The parallelized version was developed about 6 years later and its first formulation can be found in Raasch and Schröter (2001). Therewith, PALM was one of the first parallelized LES models for atmospheric research at all. Many people have helped in developing the code further over the past 15 years, and large parts of the code have been added, optimized and improved since then. For example, embedded models such as a Lagrangian cloud model (LCM) as part of a Lagrangian particle model (LPM) and a canopy model have been implemented. Also, an option for Cartesian topography is available. Moreover, the original purpose of the model to study atmospheric turbulence was extended by an option for oceanic flows. Thus, Raasch and Schröter (2001) can no longer be considered an adequate reference for current and future research articles [6,7].

\section{PROPOSED SCHEME}

\subsection{Lagrangian Droplet Tracking Algorithm}

Particle-tracking methods are widely used in fluid mechanics, multi-target tracking research and ocean flows because of their unique ability to reconstruct long trajectories with high spatial and temporal resolution. Researchers have recently demonstrated 3D tracking of several objects in real time, but as the number of objects is increased, real-time tracking becomes impossible due to data transfer and processing bottlenecks. This problem may be solved by using parallel processing. Lagrangian particle tracking (LPT) has become widely used in experimental fluid dynamics. In an LPT experiment, the flow of interest is seeded with tracer particles that are then imaged to reconstruct the fluid motion. While the related Eulerian measurement technique of particle image velocimetry (PIV) commonly uses a very high seeding density and calculates average velocity vectors for clusters of particles based on the assumption that nearby particles move similarly, LPT uses a lower seeding density but finds individual, longer particle tracks that may be used to calculate both Eulerian and Lagrangian quantities. The first task of an LPT system is to process the images from the cameras to yield the positions of the tracer particles in the image space of each camera. After finding the two dimensional particle positions, the second task is to reconstruct the three-dimensional coordinates, since each camera images only a two-dimensional projection of the measurement volume [8, 12].

Lagrangian particles can be released in prescribed source volumes at different points in time. The particles then obey: 


$$
\frac{\mathrm{dx}_{\mathrm{p}, \mathrm{i}}}{\mathrm{dt}}=\mathrm{u}_{\mathrm{p}, \mathrm{i}}(\mathrm{t})
$$

Where $\mathrm{x}_{\mathrm{p}, \mathrm{i}}$ describes the particle location in $\mathrm{x}_{\mathrm{i}}$ direction $(\mathrm{i} \in\{1,2,3\})$ and $\mathrm{u}_{\mathrm{p}, \mathrm{i}}$ is the respective velocity component of the particle. Particle trajectories are calculated by means of the turbulent flow fields provided by PALM for each time step. The location of a certain particle at time $t+\Delta t_{L}$ is calculated by

$$
x_{p, i}\left(x_{p s, i} t+\Delta t_{l}\right)=x_{p, i}\left(x_{p s, i}, t\right)+\int_{t}^{t+\Delta t l} u_{p, i}(t) d t
$$

Where, $x_{\mathrm{p}, \mathrm{i}}$ is the spatial coordinate of the particle source point and $\Delta \mathrm{t}_{\mathrm{L}}$ is the applied time step in the Lagrangian particle model. Note that the latter is not necessarily equal to the time step of the LES model. The integral in the Eq. above is evaluated using either a Runge-Kutta (2nd- or 3rd-order) or the (1st-order) Euler time-stepping scheme.

The velocity of a weightless particle that is transported passively by the flow is determined by

$$
u_{p, i}=u_{i}\left(x_{p, i}\right)
$$

And for non-passive particles (e.g., cloud droplets) by:

$$
\frac{d x_{p, i}}{d t}=\frac{1}{\tau_{p}}\left(u_{i}\left(x_{p, i}\right)-u_{p, i}\right)-\delta_{i 3}\left(1-\frac{\rho_{0}}{\rho_{p, 0}}\right) g
$$

Where, $\tau_{p}$ is the Stokes drag relaxation time scale, $\rho_{0}$ is the density of air and $\rho_{p, 0}$ is the density of particle $[11,10]$.

\subsection{Storing Lagrangian Particle droplet}

In order to handle an extremely large number of droplets in a cloud, the concept of a super-droplet is introduced. Each super-droplet represents a large number of real droplets of a given size. Handling hundreds of millions of particles, efficient storing is essential for a good performance Most applications demand particles located at a certain location (e. g., collision process is computed for all particles located in a certain grid box). Sorting the particles by their respective grid-box increases the computability of the code, but needs time for the sorting itself [9].

\section{CONCLUSION}

This paper represents the unsteady nature of LES and the variety of models (such as WRF with LES and PALM with LES) present needs more computational requirements than classical turbulence models. LES has been developed since the 1960 's, but using powerful computational tools such as high speed computers have only recently been available.

As mentioned, using LES turbulence can be simulated with an intensive care and the reason is that this method is very sensitive to the even smallest variation in some computational parameters. Defining constants and choosing the appropriate procedure should be done with caution. There is a need for a system of fine grids, In order to gain reliable results with LES, which leads to massive amount of data and time of computation. It should be noted that the treatment of unstructured grids in LES has started recently and it is a new field of research.

\section{REFERENCES}

[1] Taghinia, Javad. "Large Eddy Simulation Method-An Introduction." (2011).

[2] Mirocha, J. D., et al. "New Large Eddy Simulation Subfilter Turbulence Models Implemented Into the Advanced Research WRF Version 3.0.".

[3] Riechelmann, T., Y. Noh, and S. Raasch. "A new method for large-eddy simulations of clouds with Lagrangian droplets including the effects of turbulent collision." New Journal of Physics 14.6 (2012): 065008.

[4] Yang Zhiyin,"Large-eddy simulation: Past, present and the future",Chinese Journal of Aeronautics Volume 28, Issue 1.

[5] Sullivan, Peter P.; McWilliams, James C.; Moeng, Chin-Hoh (1994). "A subgrid-scale model for large-eddy simulation of planetary boundarylayer flows". Boundary-Layer Meteorology. Kluwer Academic Publishers. 71 (3): 247-276.

[6] Javad Taghinia,"A Brief Review Of The Large Eddy Simulation Method", Aalto University, Department of Applied Mechanics.

[7] Raasch, Siegfried, and Michael Schröter. "PALM - a large-eddy simulation model performing on massively parallel computers." Meteorologische Zeitschrift 10.5 (2001): 363-372.

[8] Raasch, S. and Etling, D.: Numerical simulation of rotating turbulent thermal convection, Beitr. Phys. Atmos., 64, 185-199, 1991

[9] S. Raasch,B. Maronga et. al "The Parallelized Large-Eddy Simulation Model (PALM) version 4.0 for atmospheric and oceanic flows: model formulation, recent developments, and future perspectives", Geosci. Model Dev., 8, 2515-2551, 2015.

[10] C-H Moeng and PP Sullivan,"Large-Eddy Simulation”,Encyclopedia of Atmospheric Sciences $2^{\text {nd }}$ Edition, Volume 4.

[11] Cuijpers, J. W. M. and Duynkerke, P. G.: Large-eddy simulation of trade-wind cumulus clouds, J. Atmos. Sci., 55, 151-162, doi:10.1175/15200469(1998).

[12] https://palm.muk.uni-hannover.de/trac.

\section{BIOGRAPHY}

Sachin H. Shinde is currently pursuing M. Tech degree in Computer Science and Engineering (Specialization in Information Technology) Walchand College of Engineering, Sangli, Maharashtra, India - 415416. His research area are High Performance Computing, Cloud Simulation Models. 